\title{
Asymmetry Induced Localization
}

\author{
H. KOHLER*
}

Instituto de Ciencia de Materiales de Madrid, CSIC, Sor Juana de la Cruz 3, Cantoblanco, 28049 Madrid, Spain

\begin{abstract}
We consider a two-level system, which couples via non-commuting operators to two independent oscillator baths. When the coupling is symmetric, the renormalized hopping matrix element is finite even for infinitely strong coupling strength. The two-level system is in a delocalized phase. For finite coupling strength a localization transition occurs for a critical asymmetry angle, which separates the localized from the delocalized phase. Using the method of flow equations we are able to monitor real time dynamics.
\end{abstract}

DOI: 10.12693/APhysPolA.124.1053

PACS: 05.30.-d, 03.65.Yz, 05.45.Mt, 73.23.-b

\section{Introduction}

Localization phenomena are ubiquitous in quantum physics. They are at the core of our understanding how classical physics comes about in quantum mechanics. The Anderson localization, respectively weak localization is a purely wave-mechanical effect, where extended waves are suppressed by a subtle interplay between disorder and coherent wave propagation [1-3]. Decoherence, the loss of quantum coherence by interaction with environmental degrees of freedom, leads therefore unavoidably to a suppression of the Anderson localization. On the other hand, decoherence itself is the key ingredient for the quantum measurement process, which is under certain circumstances itself a localization phenomenon.

A major objective of this manuscript is to understand the last assertion in more detail. To this end let us first consider a rather generic model of decoherence, given by the following system bath Hamiltonian:

$$
\hat{H}=\hat{H}_{\mathrm{S}}+\hat{A}_{\mathrm{S}} \otimes \hat{E}_{\text {env }}+\hat{H}_{\mathrm{env}}
$$

where $\hat{A}_{\mathrm{S}}$ is an arbitrary operator of the system and $\hat{E}_{\text {env }}$ is an operator of the environment. Following Zurek [4] (see $[5,6]$ for reviews) the operator $\hat{A}_{\mathrm{S}}$ singles out a preferred basis, the so-called pointer basis, for the reduced density matrix $\rho_{\mathrm{S}}$ of the system. In this basis the operator $\hat{A}_{\mathrm{S}}$ is diagonal. If the total density matrix is initially in a product state and interaction is switched on, the system becomes entangled with the environment on a time scale, which is called decoherence time. If the coupling to the environment is strong enough in the long time limit the reduced density matrix is almost diagonal in the pointer-basis. The question, whether or not an environment efficiently spoils quantum coherence or not, is subtle and cannot be answered generally, but must be investigated case by case.

For this reason we consider as specific example a particle in a double well potential as depicted in Fig. 1. Examples of this situation include some types of chemical reactions or the motion of a magnetic flux trapped in a radio-frequency SQUID ring for external flux bias near

\footnotetext{
*e-mail: hkohler@icmm.csic.es
}

half a flux quantum [7]. In Fig. 1 the ground state wave function is sketched by the red curve. It has two peaks localized at the minima of the potential, whose width is determined by the tunneling rate respectively by the oscillator frequency $\omega_{0}$ in each well. Following the above arguments a coupling of the particles position to the environment sharpens the peak, i.e. increases its height and decreases its width. The question, if and under what conditions the environment is capable to shrink the peak to a delta, i.e. to fully diagonalize $\rho_{\mathrm{S}}$ in position was subject to a large number of investigations during the last decades. Let us briefly compile the most notable facts $[6,8-10]$.

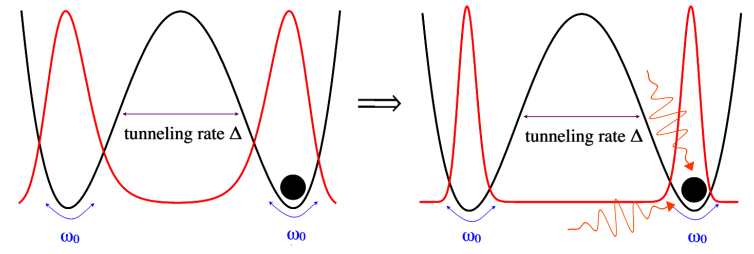

Fig. 1. Sketch of the physical situation of the dissipative two-level system. On the right, the interaction of the particle with a Bosonic environment (sketched by photonic lines) gives rise to a sharpening of the peaks.

To begin with one must assert that the position expectation value will always have a non-zero variance, since position is a continuous degree of freedom. However in the present context we are not interested in fluctuations which take place in one and the same well, but rather in the fluctuations due to tunnelling. In the limit where the oscillator frequency is much larger than the tunnelling rate $\omega_{0} \gg \Delta$ intra-well fluctuations are more and more suppressed and the double well potential described by a two-level system [8]. If further the environment is modelled by a bath of harmonic oscillators, the dynamics is described by the spin boson Hamiltonian or dissipative two-level system $(\hbar=1)$ :

$$
\begin{aligned}
& \hat{H}=\hat{H}_{0}+\hat{H}_{\mathrm{I}}, \\
& \hat{H}_{0}=-\Delta \hat{S}_{x}+\sum_{k} \lambda_{k}\left(\hat{a}_{k}+\hat{a}_{k}^{\dagger}\right) \otimes \hat{S}_{z}+\sum_{k} \omega_{k} \hat{a}_{k}^{\dagger} \hat{a}_{k},
\end{aligned}
$$

where $\hat{S}_{n}, n=x, y, z$ are spin operators and $\hat{a}_{k}$ are 
Bosonic annihilation operators. It is a well-known fact [9] that the complete information about the effect of the environment is encapsulated in a single spectral function $J(\omega)=\sum_{k} \lambda_{k}^{2} \delta\left(\omega-\omega_{k}\right)$. Moreover, it has become standard to classify the oscillator bath according to the power law behavior of $J(\omega)=\omega^{s}$ at zero frequency into subohmic $(s<1)$, ohmic $(s=1)$ and superohmic $(s>1)$. In order to avoid ultraviolet singularities the spectral function must be equipped with a high frequency cutoff $\omega_{\mathrm{c}}$. For an ohmic bath $J(\omega)=2 \gamma \omega$, and the dynamics of the spin depends on the value of the dimensionless parameter $\gamma$. For $\gamma<1 / 2$ the system is in the weak coupling regime, characterized by underdamped Rabi oscillations of ground state expectation values and correlation functions of the spin observables. At $\gamma=1 / 2$ a crossover takes place from underdamped to overdamped oscillations. Formally, the Rabi frequency becomes infinite. However, the effective tunneling rate is still non-zero. This crossover is in nature very similar to the crossover from underdamped to overdamped oscillations of the harmonic oscillator. Finally, for $\gamma=1$ the effective tunnelling rate becomes strictly zero. The reduced density matrix of the spin is $\rho_{\mathrm{S}}=\frac{1}{2} \mathbb{1}_{2}$. This means we have a twofold degenerate ground state, one with the particle localized in the left and one with the particle localized in the right well. The two ground states clearly break the $\mathbb{Z}_{2}$ parity-symmetry of the system and are separated by an infinite energy barrier. Thus the criteria of a quantum-phase transition are met. The phase transition is smooth in the thermodynamical quantities and is therefore of the Kosterlitz-Thouless (KT) type.

The quantum phase transition at $\gamma=1$ which separates a localized phase from a delocalized phase, is an extreme case of decoherence. The reduced density matrix of the spin is strictly diagonal in the pointer basis. Thus in the language of quantum measurement theory the Bosonic modes provide a complete measurement of the $\hat{S}_{z}$ component of the spin, which behaves like a classical binary random variable.

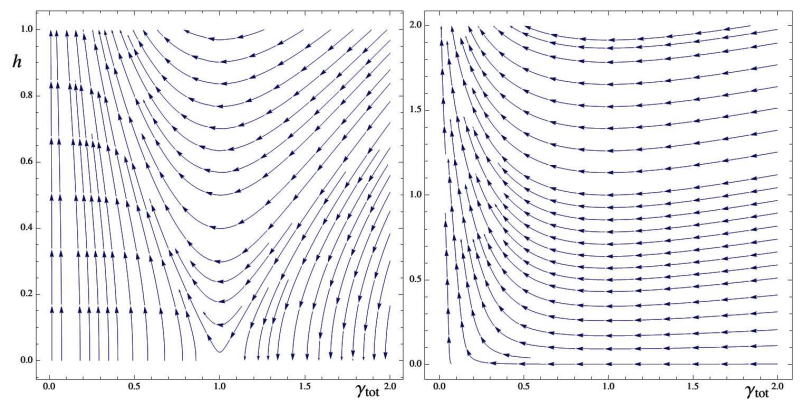

Fig. 2. On the left RNG-flow in the $\gamma_{\text {tot }}-h$ plane for the asymmetry angle $\theta=0$ corresponding to a single baths TLS. The flow is described by Eq. (3). On the right the RNG-flow and for symmetric coupling, $\theta=\pi / 4$. The flow is described by Eq. (6) with $\theta=0$.

This phase transition was first discovered by Anderson and Yuval $[11,12]$ in a perturbative renormalization group analysis. There the small parameter is not the interaction strength but the ratio $h=\Delta / \omega_{\mathrm{c}}$. The renormalization group (RNG) equations are

$$
\frac{\mathrm{d} \gamma}{\mathrm{d} l}=-h^{2} \gamma, \quad \frac{\mathrm{d} h}{\mathrm{~d} l}=-(1-\gamma) h
$$

with $l=-\ln \omega_{\mathrm{c}}$. The flow is depicted on the left hand side (1.h.s.) of Fig. 2. It is seen that for $\gamma>1$ the dimensionless tunneling rate $h$ flows to zero for $\gamma>1$ and it flows to infinity for $\gamma<1$. This latter behavior is clearly unphysical. The breakdown of the RNG flow however is not surprising, since as $h$ increases the condition $h \ll 1$ under which the Eqs. (3) were derived is not fulfilled any more. The common estimate $\Delta_{\mathrm{r}} / \Delta=h^{\gamma /(1-\gamma)}$ for the renormalized tunnel matrix element was found by Silbey and Harris [13] by different methods.

\section{The 2 bath two level system (2BTLS)}

In the introductory section the Kosterlitz-Thouless localization phase transition in the spin-boson model was physically interpreted as a maximal efficient measurement of the particle's position. With respect to this interpretation a natural question arises: If in the spin boson model the $\hat{S}_{z}$ component is measured by the oscillator bath. What happens if a second bath couples to a different spin operator $\hat{S}_{y}$ ? Since the two environments simultaneously attempt to measure two non-commutative observables, which is impossible due to Heisenberg's uncertainty principle, it is intuitively clear that in some form cancellations must take place.

These cancellations were indeed observed in a variety of different systems as a two-level system (TLS) coupled to two oscillator bath [14-17] or to two spin-baths [18], a harmonic oscillator coupled to two oscillator bath [19-22] in spin-lattices [23] or the Josephson networks [24]. The relation to two channel Kondo physics was already pointed out in [14, 25, 26]. In [14] the notion frustration of decoherence was coined for effects which are ascribed to the competition and mutual cancellation of two environments, which couple to non-commuting observables of a central system. In the following we review the most notable effects of quantum frustration on the example of a TLS which couples to two oscillator baths (2BTLS) and briefly touch on the quantum frustrated harmonic oscillator. The Hamiltonian of the 2BTLS is given by $(\hbar=1)$ :

$$
\begin{aligned}
& \hat{H}=\hat{H}_{0}+\hat{H}_{\mathrm{I}}, \hat{H}_{0}=-\Delta \hat{S}_{x}+\sum_{k} \omega_{k} \hat{a}_{k}^{\dagger} \hat{a}_{k}+\sum_{l} \nu_{l} \hat{b}_{l}^{\dagger} \hat{b}_{l}, \\
& \hat{H}_{\mathrm{I}}=\sum_{k} \lambda_{k}\left(\hat{a}_{k}+\hat{a}_{k}^{\dagger}\right) \otimes \hat{S}_{z}-\mathrm{i} \sum_{l} \mu_{k}\left(\hat{b}_{l}-\hat{b}_{l}^{\dagger}\right) \otimes \hat{S}_{y} .
\end{aligned}
$$

As in the case of a single bath two-level system we focus on ohmic baths. The two baths are described by two dimensionless coupling constants $\gamma_{a}$ and $\gamma_{b}$ and by a cutoff frequency $\omega_{\mathrm{c}}$. In view of the foregoing discussion it is useful to introduce a total coupling parameter and an asymmetry angle according to

$$
\gamma_{\text {tot }}=\gamma_{a}+\gamma_{b}, \quad \theta=\arctan \sqrt{\gamma_{b} / \gamma_{a}},
$$

such that $\theta=\pi / 4$ if the spin couples with the same cou- 
pling strength to both bath and $\theta=0$ if the spin couples tto a single bath. The KT phase transition mentioned in the introduction takes place at the point $(1,0)$ in the $\gamma_{\text {tot }}-\theta$ plane. In Ref. [14] the RNG equation corresponding to Eqs. (3) were derived for the 2BTLS for general $\gamma_{\text {tot }}$ and $\theta$. We state them here in terms of the asymmetry angle $\theta$ and the total coupling strength $\gamma_{\text {tot }}$ :

$$
\begin{aligned}
& \frac{\mathrm{d} \theta}{\mathrm{d} l}=\frac{\gamma_{\mathrm{tot}}}{2}[1-\sin (2 \theta)-\cos (2 \theta)], \\
& \frac{\mathrm{d} \gamma_{\mathrm{tot}}}{\mathrm{d} l}=-\gamma_{\mathrm{tot}}^{2} \sin ^{2}(2 \theta)-\gamma_{\mathrm{tot}} h^{2}, \\
& \frac{\mathrm{d} h}{\mathrm{~d} l}=\left(1-\gamma_{\mathrm{tot}}\right) h .
\end{aligned}
$$

For $\gamma_{\text {tot }} \neq 0$ the flow of $\theta$ has two fixed points at $\theta=0$ and at $\theta=\pi / 4$. The fixed point at $\theta=0$ is stable, the fixed point at $\theta=\pi / 4$ is unstable. For $\theta=0$ the $R N G$ equations for $\gamma_{\text {tot }}$ and $h=\Delta / \omega_{\text {c }}$ reduce to Eq. (3).

In Fig. 2 the RNG flow is plotted for $\theta=0$ and for $\theta=\pi / 4$. Whereas for a single bath $(\theta=0) h$ flows to zero for $\gamma_{\text {tot }}>1$ and to infinity for $\gamma_{\text {tot }}<1$ for symmetric coupling $h$ flows to infinity for arbitrary $\gamma_{\text {tot }}$. Thus at the symmetric point no quantum-phase transition occurs for arbitrary strong finite overall coupling strength. This is in accordance with the physical interpretation that two observers cannot measure two non-commuting observables at the same time. This is by now the most striking signature of quantum frustration. The question, whether for an arbitrary asymmetry angle $0 \leq \theta \leq \pi / 4$ a phase transition occurs and for what $\gamma_{\text {tot }}$ is delicate and cannot be answered by an analysis of the fixed point manifold of Eqs. (6), for one reason because, as mentioned before, these equations do not yield the correct weak coupling fixed point for $h$.

In Refs. [14, 15] equilibrium correlation functions were calculated by a numerical renormalization group method. Also these quantities feature signatures of quantum frustration. As pointed out in the introductory section in the single bath spin boson model the equilibrium correlation functions exhibit a crossover from overdamped to underdamped oscillation at $\gamma=1 / 2$. This crossover can be seen in the behavior of the transverse susceptibility

$$
\chi_{z z}(\omega)=-\mathrm{i} \int_{0}^{\infty} \frac{\mathrm{d} t}{2 \pi} \mathrm{e}^{\mathrm{i} \omega t}\left\langle\left[S_{z}(0), S_{z}(t)\right]\right\rangle,
$$

respectively of its imaginary part $\chi_{z z}=\chi_{z z}^{\prime}+\mathrm{i} \chi_{z z}^{\prime \prime}$. For vanishing coupling strength $\gamma=0$ the quantity $\chi_{z z}^{\prime \prime} / \omega$ exhibits a delta peak at bare tunneling rate $\Delta$. As $\gamma$ increases this peak broadens and the maximum moves at $\Delta_{\mathrm{r}}$ towards lower frequencies. Finally, at the crossover $\gamma=1 / 2$ the peak at $\Delta_{\mathrm{r}}$ disappears completely and the function has its maximum at $\omega=0$.

In Fig. 3 the function $\chi_{z z}^{\prime \prime} / \omega$ is plotted for $\theta=\pi / 4$ for several values of $\gamma_{\text {tot }}$. As expected the peak broadens however it never disappears even for values of $\gamma_{\text {tot }}>1 / 2$. This means that the crossover from overdamped to underdamped is absent for symmetric coupling, which is another signature of quantum frustration. Both parts in Fig. 3 show the same quantity, obtained by different
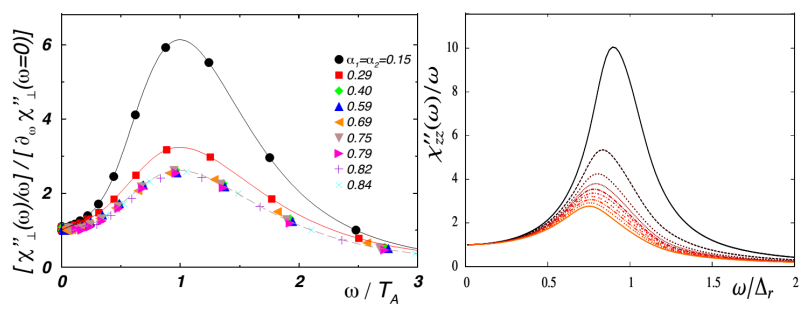

Fig. 3. Imaginary part of the transverse suceptibility $\chi^{\prime \prime}(\omega)$ for different values of the overall coupling. The figure on the 1.h.s. is obtained from a numerical renormalization group analysis. It is taken from Ref. [15]. The figure on the r.h.s. is obtained from the flow equation approach. It is taken from Ref. [17]. The values for $\gamma_{\text {tot }}$ are $\gamma_{\text {tot }}=0.1 \sqrt{2} n, 1 \leq n \leq 10$, from top to bottom (online color: from dark-colored to light-colored). The number of bath modes is $400, \Delta / \omega_{\mathrm{c}}=1 / 10$.

methods, see end of Sect. 4 .

In Refs. [19-21] the dissipative harmonic oscillator was investigated, where the central harmonic oscillator couples with position and momentum to two independent oscillator baths. The Hamiltonian is obtained from Eq. (4) by the replacement $\hat{S}_{z} \rightarrow \hat{q}, \hat{S}_{y} \rightarrow \hat{p}$ and $\hat{S}_{x} \rightarrow \frac{1}{2}\left(\hat{q}^{2}+\hat{p}^{2}\right)$. This substitution is physically equivalent to a replacement of the two level system by an $N$-level system and taking the limit $N$ to infinity. Large spins occur for instance in magnetic particles [27].

For the harmonic oscillator signatures of quantum frustration were found albeit in a weaker form than for the 2BTLS: the crossover from underdamped to overdamped equilibrium oscillations which occurs for a single bath at coupling strength $\gamma=1$ never occurs for symmetric coupling.

Since the system is exactly solvable also relaxation to equilibrium could be studied rigorously [20]. For a decoupled initial state the expectation values of the central oscillator incur important initial slips on the time scale of the inverse cutoff frequency [28] causing an almost complete decay of coherence on this time scale. These initial slips become more pronounced for a coupling to two baths leading to the conclusion that no features of quantum frustration can be observed in the relaxation of the dissipative harmonic oscillator. The non-equilibrium dynamics of the 2BTLS will be addressed in Sect. 5 .

\section{Hamiltonian flow equations}

A modern method to investigate the 2BTLS is the method of Hamiltonian flow equations. This method was developed in the beginning of the nineties by Glazek and Wilson [29] and by Wegner [30]. It provides a well controlled way to approximately calculate the spectrum of a Hamiltonian, which can in principle be arbitrarily complicated. It is similar in spirit of a renormalization group transformation. But, whereas renormalization group methods are mainly designed to approximate the spectrum in the long wavelength sector, the flow equations yield approximate results for the total spectrum. 
We outline the method following mainly the ideas of Wegner [30, 31].

Let us consider an equivalence class of Hamiltonians which are related via a one-parameter unitary transformation to the original one

$$
\hat{H}(l)=\hat{U}(l) \hat{H} \hat{U}^{\dagger}(l) .
$$

The parameter $l$ which defines the unitary transformation is called flow parameter. The derivative of this equation with respect to the flow parameter yields the flow equations

$$
\frac{\mathrm{d} \hat{H}(l)}{\mathrm{d} l}=[\hat{\eta}(l), \hat{H}(l)],
$$

where $\hat{\eta}(l)=-\hat{U}(l) \frac{\mathrm{d}}{\mathrm{d} l} \hat{U}^{\dagger}(l)$ is the generator of the flow. The generator is chosen such that the Hamiltonian $\hat{H}(\infty)$ obtains a particular simple form. Wegner's choice, which will be called canonical generator in the following is $\hat{\eta}=\left[\hat{H}_{0}, \hat{H}\right]$, where $\hat{H}_{0}$ is the non-interacting part of the full Hamiltonian. This choice for $\hat{H}_{0}$ is not mandatory but convenient. In principle other choices are possible. ${ }^{\dagger}$ To see why the canonical generator is so convenient it suffices to look at the evolution of $\hat{H}_{\mathrm{I}}=\hat{H}-\hat{H}_{0}$. For simplicity we assume $\hat{H}$ to be traceclass, then $F=\operatorname{Tr}\left(\hat{H}_{\mathrm{I}}\right)^{2}$ is well defined and obeys the flow equation

$$
\frac{\mathrm{d} F(l)}{\mathrm{d} l}=-2 \sum_{k, k^{\prime}}\left(\varepsilon_{k}-\varepsilon_{k^{\prime}}\right)^{2}\left|\hat{H}_{k k^{\prime}}\right|^{2},
$$

where $\varepsilon_{k}$ are the eigenvalues of $\hat{H}_{0}$. If $\hat{H}_{0}$ is non-degenerate the right hand side is always smaller than one and consequently $\lim _{l \rightarrow \infty} F(l)=0$. Therefore $\hat{H}(\infty)=$ $\hat{H}_{0}(\infty)$, or in other words the initial Hamiltonian $\hat{H}(0)$ was diagonalized.

Maybe the best way to understand the idea of the method is to work it out in the most simple example. To this end we assume the Hamiltonian to be just a $2 \times 2$ matrix $\hat{H}=E \hat{S}_{z}+V \hat{S}_{x}$, which has obviously eigenvalues $E_{ \pm}= \pm \sqrt{E^{2}+V^{2}} / 2$. Let us see how this result comes about within the flow equation approach. First the commutator of the diagonal part of the Hamiltonian, in this case $\hat{H}_{0}=E \hat{S}_{z}$, with the full Hamiltonian is calculated. It yields the canonical generator $\hat{\eta}=\left[\hat{H}_{0}, \hat{H}\right]=\mathrm{i} E V \hat{S}_{y}$. Then the flow equation is

$$
\frac{\mathrm{d} \hat{H}(l)}{\mathrm{d} l}=[\hat{\eta}(l), \hat{H}(l)]=V^{2}(l) E(l) \hat{S}_{z}-V(l) E^{2}(l) \hat{S}_{x},
$$

which yields two coupled non-linear differential equations for the two parameters $E(l)$ and $V(l)$ :

$$
\frac{\mathrm{d} E(l)}{\mathrm{d} l}=V^{2}(l) E(l), \quad \frac{\mathrm{d} V(l)}{\mathrm{d} l}=-V(l) E^{2}(l) .
$$

Although these equations can be solved, this is not necessary, since we need to know only the values of $E(\infty)$ and $V(\infty)$. Observing that $E^{2}(l)+V^{2}(l)$ is constant un-

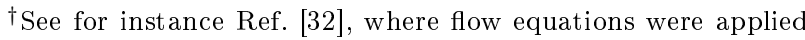
to the single bath spin boson model. In this work not the free system served as $\hat{H}_{0}$ but the interacting one at the Toulouse point $\gamma=1 / 2$, where the system is integrable. der the flow $E^{2}(\infty)=E^{2}+V^{2}$, which is the exact result, if $V(\infty)=0$. An explicit calculation yields indeed

$$
V(l)=\frac{V^{4}(0)+E^{2}(0) V^{2}(0)}{V^{2}(0)+E^{2}(0) \mathrm{e}^{2 l\left(E^{2}+V^{2}\right)}} .
$$

This means that $V(\infty)$ is indeed zero as long $E \neq 0$. This simple example demonstrates that Hamiltonian flow equations yield exact results as long as no further approximations are made. However, Eq. (11) is special in the sense that on the l.h.s. and the r.h.s. the same spin operators appear, allowing for a closed set of differential equations for the coefficients. For a general interacting many-body Hamiltonian the commutator on the r.h.s. of Eq. (9) creates interaction terms not present in the original Hamiltonian. In principle, one has two options to overcome this problem. One can either include the newly generated terms right from the outset in a more general original Hamiltonian. This leads unavoidably to a larger and larger number of parameters and thus to a larger and larger number of ODE's to be solved. The second option consists in neglecting certain terms created by the commutator. In practice one employs a combination of both options.

In the case of the 2BTLS Hamiltonian it is readily seen that the double commutator $\left[\left[\hat{H}_{0}, \hat{H}\right], \hat{H}\right]$ creates new interaction terms. On the one hand, these are terms proportional to $\hat{S}_{z} \otimes \hat{b}_{l}$ and i $\hat{S}_{y} \otimes \hat{a}_{k}$, respectively, to their Hermitian conjugates. On the other hand, there arise terms proportional to $\hat{S}_{x} \otimes \hat{c}_{k} \hat{c}_{k^{\prime}}$, where the operator $\hat{c}$ can be any of the creation and annihilation operators $\hat{a}^{\dagger}, \hat{b}^{\dagger}, \hat{a}, \hat{b}$. These terms are included into an initial $\hat{H}$ with a more general interaction part

$$
\begin{aligned}
& \hat{H}_{\mathrm{I}}^{\mathrm{enh}}=\frac{1}{2} \hat{H}_{\mathrm{I}}-\mathrm{i} \sum_{k} \alpha_{k} \hat{S}_{y} \otimes \hat{a}_{k}+\sum_{l} \beta_{l} \hat{S}_{z} \otimes \hat{b}_{l} \\
& \quad+\hat{S}_{x} \otimes \sum_{k, l}\left(s_{k l} \hat{a}_{k}^{\dagger} \hat{b}_{l}+t_{k l} \hat{a}_{k} \hat{b}_{l}\right) \\
& \quad+\hat{S}_{x} \otimes \sum_{k, k^{\prime}}\left(\sigma_{k k^{\prime}}^{(a)} \hat{a}_{k}^{\dagger} \hat{a}_{k^{\prime}}+\tau_{k k^{\prime}}^{(a)} \hat{a}_{k} \hat{a}_{k^{\prime}}\right) \\
& \quad+\hat{S}_{x} \otimes \sum_{l, l^{\prime}}\left(\sigma_{l l^{\prime}}^{(b)} \hat{b}_{l}^{\dagger} \hat{b}_{l^{\prime}}+\tau_{l l^{\prime}}^{(b)} \hat{b}_{l} \hat{b}_{l^{\prime}}\right)+\text { H.c. }
\end{aligned}
$$

The canonical generator and the double commutator $\left[\left[\hat{H}_{0}, \hat{H}\right], \hat{H}\right]$ must now be calculated with the enhanced interaction term (14). The double commutator yields terms which contain three or four creation or annihilation operators. These terms are not included in a yet more general initial Hamiltonian but are replaced by their expectation value with respect to the non-interacting ground state. Thereby a closed set of coupled ODE's is obtained for the altogether $1+4 N+2 N(4 N+1)$ parameters $\Delta, \alpha_{k}, \beta_{l}, \lambda_{k}, \mu_{l}, \sigma_{k k^{\prime}}^{(a)}, \sigma_{l l^{\prime}}^{(b)}, \tau_{k k^{\prime}}^{(a)}, \tau_{l l^{\prime}}^{(b)}, s_{k l}$ and $t_{k l}$. Here $N$ is the number of bath modes which is assumed to be the same for both baths. Let us note that in the approximation we used to close the equations, the frequencies of the bath modes $\omega_{k}$ and $\nu_{l}$ do not change under the flow. Typical values for the number of bath modes, which are feasible without too much numerical effort, lie between $N=500$ and $N=1000$. Although the flow 
equation can sometimes be treated analytically $[17,33-$ $35]$, the large number of ODE's to be solved renders the method of flow equations essentially a numerical method. A comprehensive account on Hamiltonian flow equations in many-body physics was recently given by Kehrein [35].

\section{Equilibrium properties}

The flow equations for the 2BTLS are solved numerically by an adaptive step-size fourth order Runge-Kutta algorithm. It is numerically checked that all parameters of $\hat{H}_{\mathrm{I}}^{\mathrm{enh}}(l)$ are zero at the endpoint of the integration. ${ }^{\ddagger}$ Thus that

$$
\hat{H}(\infty)=-\Delta(\infty) \hat{S}_{x}+\sum_{k} \omega_{k} \hat{a}_{k}^{\dagger} \hat{a}_{k}+\sum_{l} \nu_{l} \hat{b}_{l}^{\dagger} \hat{b}_{l} .
$$

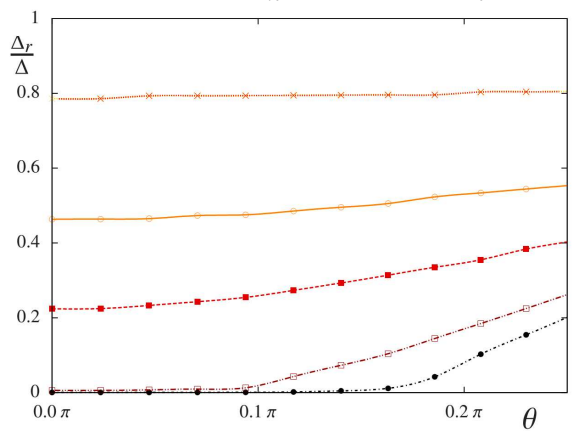

Fig. 4. Renormalized tunnelling matrix element $\Delta_{\mathrm{r}}$ as a function of the angle $\theta$ defined in Eq. (5) the main text, the total coupling strength is $\gamma_{\text {tot }}=0.1$ (crosses, yellow), $\gamma_{\text {tot }}=0.3$ (empty circles, orange), $\gamma_{\text {tot }}=0.5$ (filled boxes, red), $\gamma_{\text {tot }}=0.8$ (empty boxes, dark red) and $\gamma_{\text {tot }}=1$ (filled circles, full black line). The cutoff frequency is $\omega_{\mathrm{c}}=10 \Delta$. The number of bath modes is $N=1000$. Figure taken from Ref. [17].

In Fig. 4 the renormalized tunnel matrix element $\Delta_{\mathrm{r}} \equiv$ $\Delta(\infty)$ is plotted as a function of the asymmetry angle $\theta$ defined in Eq. (5) for different overall coupling $\gamma_{\text {tot }}$. Whereas for small overall coupling $\gamma_{\text {tot }}$ the renormalized tunneling matrix element $\Delta_{\mathrm{r}}$ is almost independent of $\theta$, it is nicely seen that if $\gamma_{\text {tot }}$ becomes larger $\Delta_{\mathrm{r}}$ is protected by a symmetric coupling. For $\gamma_{\text {tot }}=1, \Delta_{\mathrm{r}}$ renormalizes to zero within numerical accuracy. If the overall coupling is increased further, $\Delta_{\mathrm{r}}$ renormalizes to zero already for a finite asymmetry angle $\theta$. This allows us to plot a phase diagram in the $\gamma_{\text {tot }}-\theta$ plane where the localized phase $\left(\Delta_{\mathrm{r}}=0\right)$ is separated from the delocalized phase $\left(\Delta_{\mathrm{r}}>0\right)$ by a critical line. The phase diagram is plotted in Fig. 5. The critical line crosses the abscissa not at $\gamma_{\text {tot }}=1$ but at about 0.85 . This offset can be systematically improved by increasing the number of modes and the endpoint of the integration. For overall couplings large than $\gamma_{\text {tot }} \approx 2.5$ the integration routine becomes

\footnotetext{
¥Actually not all parameters flow to zero, but the diagona terms $\sigma_{k k}^{(a)}$ and $\sigma_{l l}^{(b)}$ remain finite. However their values are so small that for all practical purposes they can be neglected.
}

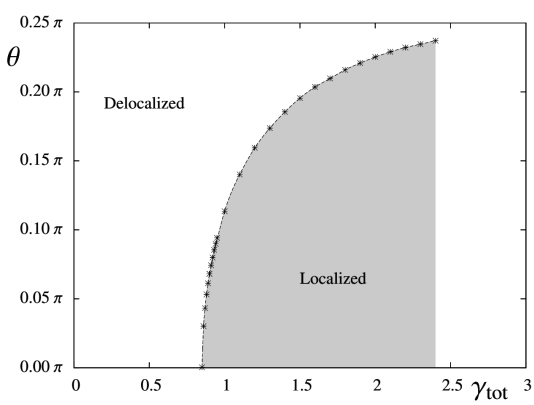

Fig. 5. Phase diagram of the 2BTLS ground state in the $\gamma_{\text {tot }}-\theta$ plane. The line indicates the critical asymmetry angle, which separates the localized from the delocalized phase. The critical angle was determined for $N=800$ bath modes. The cutoff frequency is $\omega_{\mathrm{c}}=10 \Delta$. The figure is taken from Ref. [17].

numerically unstable. Despite the numerical inaccuracies the phase diagram clearly shows that the delocalized phase is stable against small variations of the asymmetry angle $\theta$ and for small asymmetry the delocalized phase extends in a smooth fashion into the region $\gamma_{\text {tot }}>1$.

Within our approach equilibrium expectation values of an arbitrary operator can be calculated. To this end the operator is transformed by appropriate flow equations into the basis, where $\hat{H}$ is diagonal. As an example let us consider the spin operator $\hat{S}_{x}$. The operator is expanded as

$$
\begin{aligned}
& \hat{S}_{x}(l)=\frac{h_{0}}{2} \mathbb{1}_{2}+h_{x}(l) \hat{S}_{x}+S_{z} \otimes\left[\sum_{k} \chi_{k}^{(a)}\left(\hat{a}_{k}+\hat{a}_{k}^{\dagger}\right)\right. \\
& \left.+\sum_{l} \chi_{l}^{(b)}\left(\hat{b}_{l}+\hat{b}_{l}^{\dagger}\right)\right]+\mathrm{i} S_{y} \otimes\left[\sum_{k} \zeta_{k}^{(a)}\left(\hat{a}_{k}-\hat{a}_{k}^{\dagger}\right)\right. \\
& \left.\quad+\sum_{l} \zeta_{l}^{(b)}\left(\hat{b}_{l}-\hat{b}_{l}^{\dagger}\right)\right] .
\end{aligned}
$$

The commutator $\left[\hat{\eta}, \hat{S}_{x}(l)\right]$ is calculated and all terms containing two or more creation or annihilation operators are neglected. Thereby a set of ODE's is obtained for $h_{0}(l)$, $h_{x}(l), \chi_{k}^{(a)}(l), \chi_{l}^{(b)}(l), \zeta_{k}^{(a)}(l), \zeta_{l}^{(b)}(l)$, which are solved numerically. Since time evolution is trivial in the basis, where $\hat{H}$ is diagonal, an arbitrary equilibrium correlation function of $\hat{S}_{x}$ can be calculated from the knowledge of $h_{0}(\infty), h_{x}(\infty), \chi_{k}^{(a)}(\infty), \chi_{l}^{(b)}(\infty)$. The other spin operators are treated similarly. On the r.h.s. of Fig. 3 the result obtained by the flow equation method for the transverse susceptibility, defined in Eq. (7) are depicted. They agree qualitatively with the results obtained in Ref. [15] by the numerical renormalization group.

\section{Non-equilibrium: thermalization and decoherence}

Recently, the method of Hamiltonian flow equations has been extended to systems in non-equilibrium [36, 37]. The main idea of the calculation is sketched in Fig. 6. An arbitrary operator is first transformed by $\hat{U}(\infty)$ into 
the basis where $\hat{H}$ is diagonal and time evolution is trivially propagated by $\hat{H}(\infty)$. Afterwards the time evolved operator is numerically transformed back in the original basis, thereby an approximate solution of the Heisenberg equation is obtained. The expectation value of the operator with respect to an arbitrary initial state is then calculated without further problems.

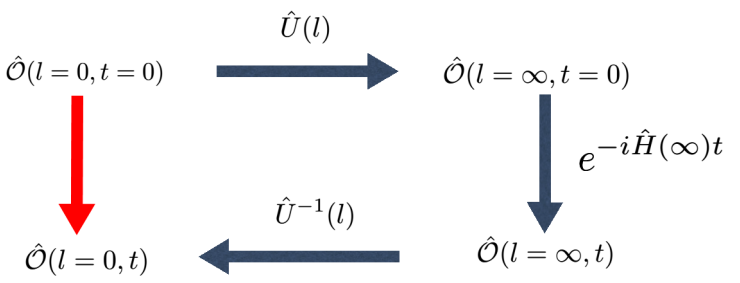

Fig. 6. Sketch of the calculation of a Heisenberg operator. First the operator is transformed into the basis where $\hat{H}$ is diagonal. Then it is propagated in time and numerically transformed back afterwards.

We apply this procedure to the decoupled non-equilibrium initial state described by the density matrix

$$
\rho_{\text {init }}=\rho_{\mathrm{S}}(0) \otimes \rho_{\mathrm{eq}}^{(a)} \otimes \rho_{\mathrm{eq}}^{(b)}, \quad \rho_{\mathrm{S}}(0)=\left(\frac{1}{2} \mathbb{1}_{2}+\hat{S}_{z}\right) .
$$

Decay of quantum coherence is monitored by the purity

$$
\mathcal{P}(t)=\frac{1}{2} \mathbb{1}_{2}+2\left\langle S_{x}(t)\right\rangle^{2}+2\left\langle S_{y}(t)\right\rangle^{2}+2\left\langle S_{z}(t)\right\rangle^{2} .
$$

Dissipation is described by the time evolution of the system Hamiltonian, i.e. the relaxation of $\left\langle\hat{S}_{x}(t)\right\rangle$ to its equilibrium value. Due to the reduced Hilbert space dimension of the system dissipation and decoherence as quantified by the purity are not independent. It is thus useful to define a parallel $\mathcal{P}_{\|}=\frac{1}{2} \mathbb{1}_{2}+2\left\langle S_{x}\right\rangle^{2}$ and a transverse purity $\mathcal{P}_{\perp}=2\left\langle S_{y}\right\rangle^{2}+\left\langle S_{z}\right\rangle^{2}$. Then $\mathcal{P}_{\|}$is associated with dissipation and $\mathcal{P}_{\perp}$ with decoherence. In the Born-Markov approximation the Bloch equations are obtained for the expectation values of the spin operators. They predict an exponential decay for both $\mathcal{P}_{\|} \propto \exp \left(-t / T_{1}\right)$ and $\mathcal{P}_{\perp} \propto \exp \left(-t / T_{2}\right)$ with decay rates $T_{1}^{-1}=2 \pi \Delta\left(\gamma_{a}+\gamma_{b}\right)$ and $T_{2}=2 T_{1}$. The decay rate shows in the Born-Markov approximation no dependence on the asymmetry angle $\theta$.

Within the Markov approximation it is possible to find higher order corrections to $T_{1}$ and $T_{2}$ the exponential decay remaining unaffected. However for small times of order of the inverse cutoff frequency the Markov approximation is not valid. A small time expansion yields for the transverse purity for symmetric coupling $(\theta=\pi / 4)$ a quadratic decay

$$
\mathcal{P}_{\perp}(t)=\frac{1}{2}-\frac{t^{2}}{4 \tau^{2}}+\mathcal{O}\left(t^{3}\right), \quad \tau^{-2}=\left(\gamma_{a}+\gamma_{b}\right) \omega_{\mathrm{c}}
$$

where $\tau$ is the so-called quantum Zeno time. On this time scale the decay of $\mathcal{P}_{\perp}$ deviates from exponential and becomes dependent of the cutoff frequency.

In Fig. 7 the transverse purity as calculated by the flow equations is plotted for symmetric coupling and for different cutoff frequencies. For small times the numerical results fit nicely the quadratic small time expansion (upper figure in Fig. 7). Moreover, one observes that the expansion is good for short time scales of order $\omega_{\mathrm{c}}^{-1}$. Afterwards, $\mathcal{P}_{\perp}$ exhibits an oscillatory crossover to the exponential decay predicted by the Bloch equations. The frequency of the oscillations scales with $\omega_{\mathrm{c}}$. The oscillations rapidly attenuate on a time scale of less than one period of the Rabi oscillations. This rather surprising oscillatory behavior of the transverse purity was to my best knowledge first reported in [17].

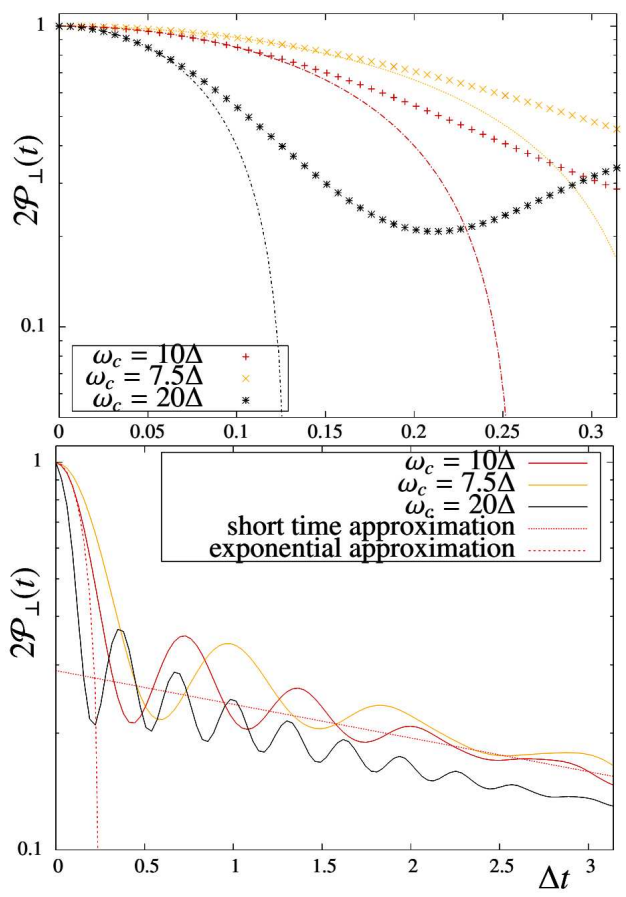

Fig. 7. Decay of the transverse purity from the initial state (17) for symmetric coupling, i.e. asymmetry angle $\theta=\pi / 4$, for three different cutoff frequencies $\omega_{\mathrm{c}}=20 \Delta$ (black), $\omega_{\mathrm{c}}=10 \Delta$ (red) and $\omega_{\mathrm{c}}=7.5 \Delta$ (yellow) on small (above) and on intermedium (below) time scales. The calculations were performed with 250 bath modes.

A more complete analysis reveals [17] that for arbitrary asymmetry angle $0 \leq \theta \leq \pi / 4$ and for a more general initial state $\rho_{\mathrm{S}}(0)=\left(\frac{1}{2} \mathbb{1}_{2}+\cos \theta^{\prime} \hat{S}_{z}+\sin \theta^{\prime} \hat{S}_{z}\right)$ the decay of $\mathcal{P}_{\perp}$ depends crucially on both angles $\theta$ and $\theta^{\prime}$ and on details of the cutoff function. Like for the quantum frustrated harmonic oscillator no universal features of quantum frustration can be identified.

\section{Summary and outlook}

Quantum frustration occurs when non-commuting observables couple to two independent heat baths. In the past several real world physical systems were proposed, which are expected to exhibit this effect. Most notably it was proposed as cooling mechanism [38]. Already in [15] for the 2BTLS the relation of quantum frustration to the Mermin-Wagner theorem [39] was pointed 
out. A similar reasoning holds for the quantum frustrated harmonic oscillator. At the symmetric point both systems have a U(1)-symmetry generated by the operator $\hat{H}_{\mathrm{S}}+\sum_{k}\left(\hat{a}_{k} b_{k}^{\dagger}+\hat{b}_{k} \hat{a}_{k}^{\dagger}\right)$, where $\hat{H}_{\mathrm{S}}=\hat{S}_{x}$ for the 2BTLS and $\hat{H}_{\mathrm{S}}=\frac{1}{2}\left(\hat{p}^{2}+\hat{q}^{2}\right)$ for the quantum frustrated harmonic oscillator. If the Hamiltonians are interpreted as one-dimensional field theories, the Mermin-Wagner theorem might be applied, which states that for low dimensional field theories a continuous symmetry cannot be broken spontaneously. Interestingly this line of arguing holds even if only a single bath is involved. As a word of caution one has to mention that the Mermin-Wagner theorem holds only for non-zero temperatures and quantum phase transitions, as considered here, are strictly speaking not forbidden.

Localization is the phenomenon, where - triggered by some mechanism - a preferred basis is singled out, in which the eigenfunctions are localized in a small region of the Hilbert space. In case of disorder induced localization this is either the position basis or for dynamical localization [40] the energy basis. For interaction induced localization it is the pointer basis. Thus it might not come as a surprise that localization is suppressed if the operator defining the preferred basis is connected to its canonical conjugate by a continuous symmetry. From this point of view it is rather surprising that inelastic scattering leads to a reduction of the localization length and not to an enhancement, since both, interaction and disorder, seem to prefer the position basis.

\section{Acknowledgments}

I acknowledge financial support from the German Research council (DFG) with grant No. Ko 3538/1-2 and from CSIC within the JAE-Doc program cofunded by the FSE (Fondo Social Europeo).

\section{References}

[1] P. Anderson, Phys. Rev. 109, 1492 (1958).

[2] P.A. Lee, T.V. Ramakrishnan, Rev. Mod. Phys. 57, 287 (1985).

[3] B. Kramer, McKinnon, Rep. Prog. Phys. 56, 1469 (1992).

[4] W.H. Zurek, Phys. Rev. D 24, 1516 (1981).

[5] J.P. Paz, W.H. Zurek, in: Coherent Matter Waves, Les Houches Session, Les Houches Summer School Series, Vol. 72, Eds. R. Kaiser, C. Westbrook, F. David, Springer, Berlin, 2001, p. 533.

[6] M. Schlosshauer, Decoherence and the Quantum to Classical Transition, Frontiers Collection Springer, Berlin 2007.

[7] A.J. Leggett, in: Percolation, Localization and Superconductivity, ASI Series B, Vol. 109, Eds. A.M. Goldman, S. Wolf, NATO, Plenum, New York 1984, p. 1.

[8] A.J. Leggett, S. Chakravarty, A.T. Dorsey, M.P.A. Fisher, A. Garg, W. Zwerger, Rev. Mod. Phys. 59, 1 (1987).
[9] U. Weiss, Quantum Dissipative Systems, 2nd ed., World Sci., Singapore 1999.

[10] H.P. Breuer, F. Petruccione, The Theory of Open Quantum Systems, Oxford University Press, Oxford 2002.

[11] P.W. Anderson, G. Yuval, J. Phys. C 4, 607 (1971).

[12] A.J. Bray, M.A. Moore, Phys. Rev. Lett. 49, 1545 (1982).

[13] R. Silbey, R.A. Harris, J. Chem. Phys. 80, 2615 (1983).

[14] A.H. Castro Neto, E. Novais, L. Borda, G. Zarand, I. Affleck, Phys. Rev. Lett. 91, 096401 (2003).

[15] E. Novais, A.H. Castro Neto, L. Borda, I. Affleck, G. Zarand, Phys. Rev. B 72, 014417 (2005).

[16] C. Guo, A. Weichselbaum, J. von Delft, M. Vojta, Phys. Rev. Lett. 108, 160401 (2012).

[17] H. Kohler, A. Hackl, S. Kehrein, Phys. Rev. B 88, 205122 (2013).

[18] D.D. Bhaktavatsala Rao, H. Kohler, F. Sols, New J. Phys. 10, 115017 (2008).

[19] H. Kohler, F. Sols, Phys. Rev. B 72, 180404 (2005).

[20] H. Kohler, F. Sols, New J. Phys. 8, 149 (2006).

[21] H. Kohler, F. Sols, Physica A 392, 1989 (2013).

[22] A. Cuccoli, N. DelSette, R. Vaia, Phys. Rev. E 81, 041110 (2010).

[23] A. Cuccoli, A. Fubini, V. Tognetti, R. Vaia, in: Path Integrals: New Trends and Perspectives, Eds. W. Janke, A. Pelster, World Sci., Singapore 2008, p. 500 .

[24] D. Giuliano, P. Sodano, New J. Phys. 10, 093023 (2008).

[25] L. Zhu, Q. Si, Phys. Rev. B 66, 024426 (2002).

[26] G. Zárand, E. Demler, Phys. Rev. B 66, 024427 (2002).

[27] E.M. Chudnovsky, J. Tejada, Macroscopic Quantum Tunneling of the Magnetic Moment, Cambridge University Press, Cambridge 1998.

[28] F. Haake, R. Reibold, Phys. Rev. A 32, 2462 (1985).

[29] S.D. Głazek, K.G. Wilson, Phys. Rev. D 48, 5863 (1993).

[30] F. Wegner, Ann. Phys. (Leipzig) 3, 77 (1994).

[31] F. Wegner, J. Phys. A 39, 8221 (2006).

[32] S. Kehrein, Nucl. Phys. B 592, 512 (2001).

[33] S. Kehrein, A. Mielke, P. Neu, Z. Phys. B 99, 269 (1996).

[34] S. Kehrein, A. Mielke, Ann. Phys. (Leipzig) 6, 90 (1997).

[35] S. Kehrein, The Flow Equation Approach to Many Particle Systems, 1st ed., Springer, Heidelberg 2006.

[36] A. Hackl, S. Kehrein, Phys. Rev. B 78, 092303 (2008).

[37] A. Hackl, S. Kehrein, J. Phys. C 21, 015601 (2009).

[38] N. Erez, G. Gordon, M. Nest, G. Kuritzki, Nature 452, 724 (2008).

[39] S. Coleman, Aspects of Symmetry, Selected Erice Lectures, Cambridge University Press, Cambridge 1985.

[40] D. Grempel, R. Prange, S. Fishman, Phys. Rev. A 29, 1639 (1984). 